\title{
Mallory bodies in alcoholic liver disease: identification of cytoplasmic filament/cell membrane and unique antigenic determinants by monoclonal antibodies
}

\author{
J A Morton, J BAStin, K A Fleming, A MCMiChaEl, J BURNS, AND \\ J O'D MCGEE*
}

From the University of Oxford, Nuffield Departments of Pathology and Medicine, John Radcliffe Hospital, Oxford

SUMMARY Polyclonal and monoclonal antibodies have been produced to Mallory body protein (MBP) extracted from isolated Mallory bodies (MBs). The polyclonal antibodies reveal a unique determinant in MBs. The first monoclonal antibody (anti-JMB1) detects a second unique determinant (JMB1) in MBs which is not detectable in normal hepatocytes. The second monoclonal antibody (anti-JMB2) shows that MBs contain another determinant which is associated with the cell and nuclear membranes and a cytoplasmic filament system of normal hepatocytes and bile duct epithelium; this antigen is not detectable in the cell membranes or cytoplasmic filament systems of hepatocytes which contain MBs. The third monoclonal antibody (anti-JMB3) reacts only with an antigen (JMB3) in mesenchymal cells of alcoholic cirrhotic liver and its significance, at this time, is unclear. It is suggested that the metabolism of JMB1, JMB2, and JMB3 antigens is deranged in hepatocytes damaged by alcohol. It is concluded that the antigenic structure of MBs is more complex than hitherto realised and that all of these antigens are distinct from prekeratin (a component of epidermal cell intermediate filaments).

Alcoholic cirrhosis is the commonest form of chronic liver disease in the Western world. ${ }^{1}$ It is generally held that only about $30 \%$ of heavy drinkers progress from acute to chronic liver disease. ${ }^{2}$ One of the hallmarks of alcohol induced liver injury is the formation of Mallory bodies (MBs). There is evidence that these intracytoplasmic hepatocyte inclusions evoke cell-mediated and humoral immune responses and these reactions have been implicated in the progression of acute alcoholic liver disease to cirrhosis. ${ }^{34}$

Isolated MBs and intermediate filaments contain proteins of similar electrophoretic mobility $;^{5}$ intermediate filaments (6-11 nm in diameter) form part of the cytoskeleton of normal hepatocytes. ${ }^{5}$ MBs also react with antisera to prekeratin, ${ }^{6}$ a component of epidermal cell intermediate filaments. It has been

*Address for correspondence: Professor J O'D McGee, University of Oxford, Nuffield Department of Pathology, John Radcliffe Hospital, Oxford, OX3 9DU

Received for publication 22 July 1980 postulated, therefore, that $\mathrm{MBs}$ result from an unspecified defect in intermediate filament metabolism in liver cells damaged by alcohol. ${ }^{6}$ More recently, it has been shown that polyclonal antibodies to Mallory body protein (MBP) raised in animals react with MBs but not with epidermis or any other tissue. ${ }^{7}$ It is evident, therefore, that MBs contain at least one specific antigen in addition to prekeratin. In this paper, using monoclonal and polyclonal antibodies to MBP, it is shown that MBs have a complex antigenic structure containing not only unique antigens but also antigens which are common to the cell membranes and cytoplasmic filaments (possibly intermediate filaments) of normal hepatocytes and bile duct epithelium. It is concluded that a derangement of the metabolism of all of these antigens occurs in hepatocytes damaged by alcohol.

\section{Methods}

The source of most reagents is detailed elsewhere. Rabbit anti-mouse IgG was prepared in this labora- 
tory and conjugated with fluorescein by standard methods. ${ }^{8}$ All tissue culture reagents and containers were purchased from Gibco-Biocult (Scotland) except Costar plates (L. H. Engineering, Bells Hill, Stoke Poges, Bucks. England).

PREPARATION OF MALLORY BODIES (MBS)

AND MALLORY BODY PROTEIN (MBP)

MBs were isolated from two alcoholic cirrhotic livers by a modification of the methods of French et al. ${ }^{9}$ and Okamura et al. ${ }^{10}$ as described earlier. ${ }^{7}$ Mallory body protein (MBP) was prepared from isolated MBs as follows: $5 \mathrm{mg}$ of isolated $\mathrm{MBs}$ in $0.5 \mathrm{ml}$ of $1 \%$ sodium dodecylsulphate containing $4 \times 10^{-3} \mathrm{M}$ dithiotheritol were heated at $60^{\circ} \mathrm{C}$ for 20 minutes; $8 \times 10^{-3} \mathrm{M}$ iodoacetamide was added, the solution heated for another 20 minutes and exhaustively dialysed against $0.01 \mathrm{M}$ phosphate buffered saline $\mathrm{pH} 7 \cdot 4$ (PBS). Isolated MBs used in all experiments were at least $95 \%$ pure as judged by electron microscopy.

\section{ANTIBODY PRODUCTION}

Specific polyclonal antibodies to MBP were produced in rabbits and characterised as previously described. ${ }^{7}$

Monoclonal antibodies were produced by the technique of Kohler and Milstein. ${ }^{11}$ Balb $\mathrm{C}$ mice were injected subcutaneously with $40 \mu \mathrm{g}$ of MBP with complete Freunds adjuvant in a final volume of $0.4 \mathrm{ml}$. Two booster injections were given at two-weekly intervals. Blood was taken from the tail vein 10 days after the last injection and tested for antibody to MBs by immunofluorescence on sections from an alcoholic liver containing MBs (see below). One of the animals which had developed antibodies was boosted again; the spleen was aseptically removed, made into a single cell suspension, and resuspended in RPMI 1640 medium supplemented with $10 \%$ fetal calf serum (FCS), penicillin $(50 \mathrm{IU} /$ $\mathrm{ml})$ and streptomycin $(50 \mathrm{IU} / \mathrm{ml}) .10^{8}$ of these cells were fused with $10^{7}$ mouse plasmacytoma cells, P3-NS1/AG4.1 (NS1), using $50 \%$ polyethylene glycol in supplemented RPMI 1640 medium. The fused cells suspended in supplemented RPMI 1640 were plated out in a volume of $1 \mathrm{ml}$ into $2 \times 48$ well Costar plates at a density of $2 \times 10^{6} / \mathrm{ml}$ and incubated for $24 \mathrm{~h}$ at $37^{\circ} \mathrm{C}$ in $5 \% \mathrm{CO}_{2} / 95 \%$ air atmosphere. One millilitre of selective medium for hybrids (RPMI $1640+10 \%$ FCS containing hypoxanthine, aminopterin, and thymidine) was added to each well. Fourteen days later the medium from each well was examined for antibody to MBs by immunofluorescence on liver sections containing MBs (see below). The wells producing antibody to
MBs had an average of 3-6 colonies per well. Each colony was harvested and grown separately in selective medium. These cultures were then cloned by dilution in microtitre plastic plates. Ten days later the colonies from the wells that received one cell in the dilution plate were assayed. Those secreting antibody were grown in large quantities in $25 \mathrm{~cm}^{2}$ Falcon flasks and the medium from these flasks (stored at $-20^{\circ} \mathrm{C}$ ) was used without additional processing as the antibody source.

\section{ABSORPTION EXPERIMENTS}

Culture media containing monoclonal antibodies were absorbed with MBs, MBP, and with a homogenate of normal liver as follows: $2 \mathrm{mg}$ isolated MBs of $95 \%$ purity $^{7}$ or $0.3 \mathrm{mg}$ MBP, or $100 \mathrm{mg}$ liver homogenate (see below) were added to three separate aliquots $(300 \mu \mathrm{l})$ of media; these mixtures were incubated at $22^{\circ} \mathrm{C}$ for two hours, centrifuged at $10,000 \times g$ for one minute, and the ability of the supernatant to react with alcoholic cirrhotic or normal liver was determined by indirect immunofluorescence. Homogenates of normal liver were prepared by homogenising 16 hour postmortem liver tissue in 3 volumes of PBS in a polytron homogeniser for 30 seconds at half power; insoluble material was recovered by centrifugation at 10,000 $\times g$ for two minutes, washed $\times 3$ in PBS and $100 \mathrm{mg}$ of this pellet was used in the absorption studies detailed above.

\section{IMMUNOFLORESCENCE}

Liver tissue obtained at necropsy (within 24 hours of death) or biopsy was frozen onto cryostat chucks in liquid nitrogen. Seven micron cryostat sections were dried at $37^{\circ} \mathrm{C}$ for one hour and fixed in acetone at $-20^{\circ} \mathrm{C}$ for 15 minutes and air dried at $22^{\circ} \mathrm{C}$. Polyclonal (diluted 1:20 in PBS) or monoclonal antibodies (undiluted) were placed on the section for one hour in a humidified atmosphere and the slide washed with PBS; fluorescein labelled sheep antirabbit IgG (diluted 1:20) or fluorescein labelled rabbit anti-mouse IgG (diluted 1:15) respectively was applied to the section for 30 minutes. After further washing in PBS, the section was mounted in $70 \%$ glycerol/PBS and examined by ultra-violet light from an HBO 200 source with incident illumination on a Leitz Ortholux microscope. Controls included incubating sections with normal rabbit serum, monoclonal antibody to HLA (A, B, and C heavy chains),${ }^{12}$ or fluorescent reagent alone.

\section{Results}

The sera of three out of four mice immunised with MBP produced antibodies which reacted with MBs 


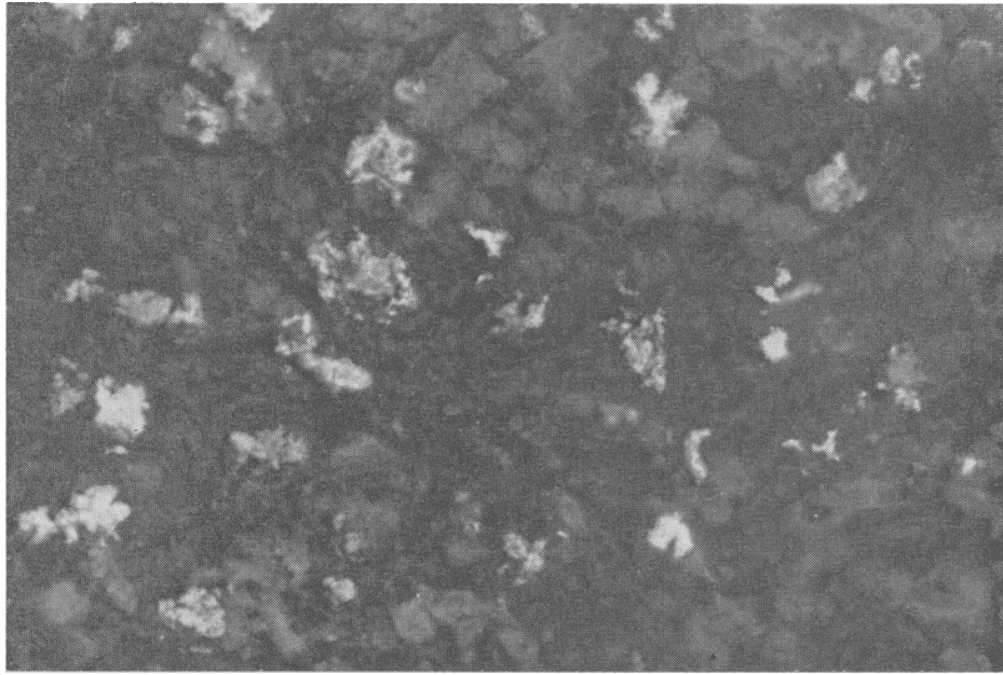

Fig. 1 Reaction of anti-JMBl with alcoholic cirrhotic liver. Only MBs are stained in this immunofluorescent preparation. $\times 400$.

in alcoholic cirrhotic liver by indirect immunofluorescence. Three cloned hybrids resulting from the fusion of spleen cells from one of these animals with mouse myeloma cells produced three monoclonal antibodies with different specificities. Antibody from one clone (designated JMB1) reacted only with MBs and no other liver constituent (Fig. 1) in alcoholic cirrhotic or normal livers. Antibody from the second clone (designated JMB2) gave a more complex pattern of staining: it reacted with MBs (Fig. 2); it labelled the plasma and nuclear membranes and a cytoplasmic filament system of morphologically normal hepatocytes in alcoholic cirrhotic liver (Fig. 2) and the same structures in hepatocytes from structurally and functionally normal livers (Fig. 3); however, it did not stain cell membranes or cytoplasmic structures other than MBs in hepatocytes which contained MBs (Fig. 2); this antibody also stained the plasma membranes and cytoplasm of interlobular bile ducts in normal and in alcoholic cirrhotic livers (Fig. 4); finally, it also labelled proliferating bile ductules in alcoholic cirrhotic liver (Fig. 5). It should be noted that, with the immunofluorescence method of detection, it is impossible to determine whether the antigen detected by the antibody produced by clone JMB2 is integral, or associated with cell membranes. Antibody from the third clone (designated JMB3) did

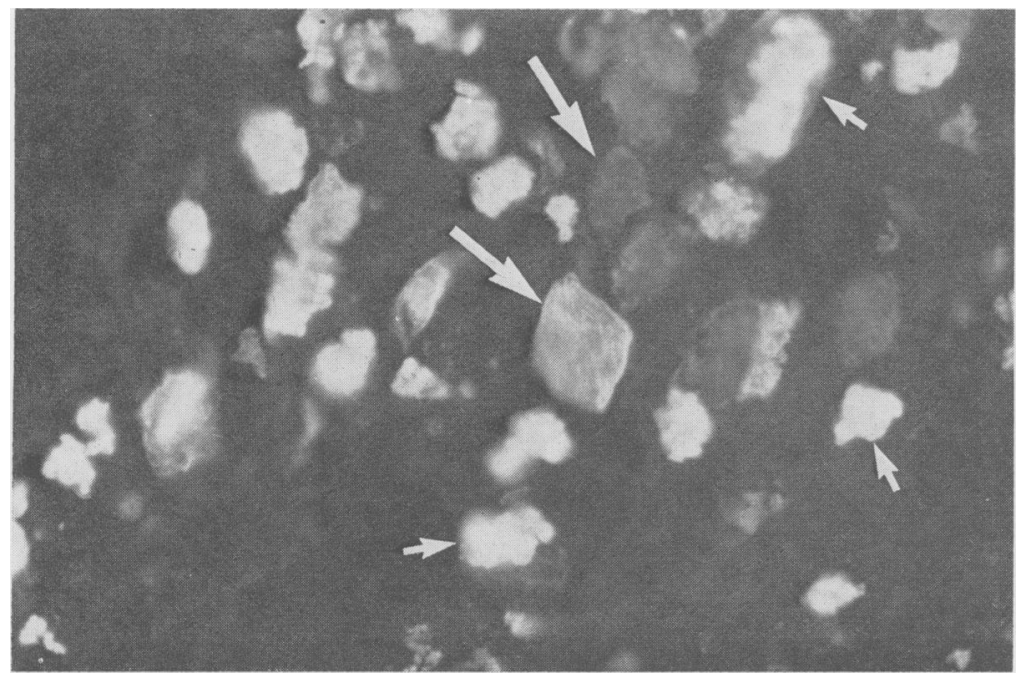

Fig. 2 Reaction of anti-JMB2 with alcoholic cirrhotic liver. $M B s$ are intensly stained (small arrows); these cells do not show membrane or cytoplasmic filament labelling in contrast with the surrounding hepatocytes (large arrows) which do not contain MBs. It should be noted that the intensity of the reaction with the latter hepatocytes is variable. $\times 400$. 
Fig. 3 Reaction of anti-JMB2 with normal liver. Plasma and nuclear membranes and a cytoplasmic filament system (arrows) within hepatocytes are stained. Sinusoidal cells are negative. $\times 400$.

Fig. 4 Reaction of anti-JMB2 with alcoholic cirrhotic liver. This monoclonal antibody also labels the cell membranes of structurally normal interlobular bile ducts. $\times 250$.

Fig. 5 Staining of proliferating bile ductules in an alcoholic cirrhotic liver. The cell membranes of the cells lining these ductules shows a strong reaction with anti-JMB2. $\times 400$.
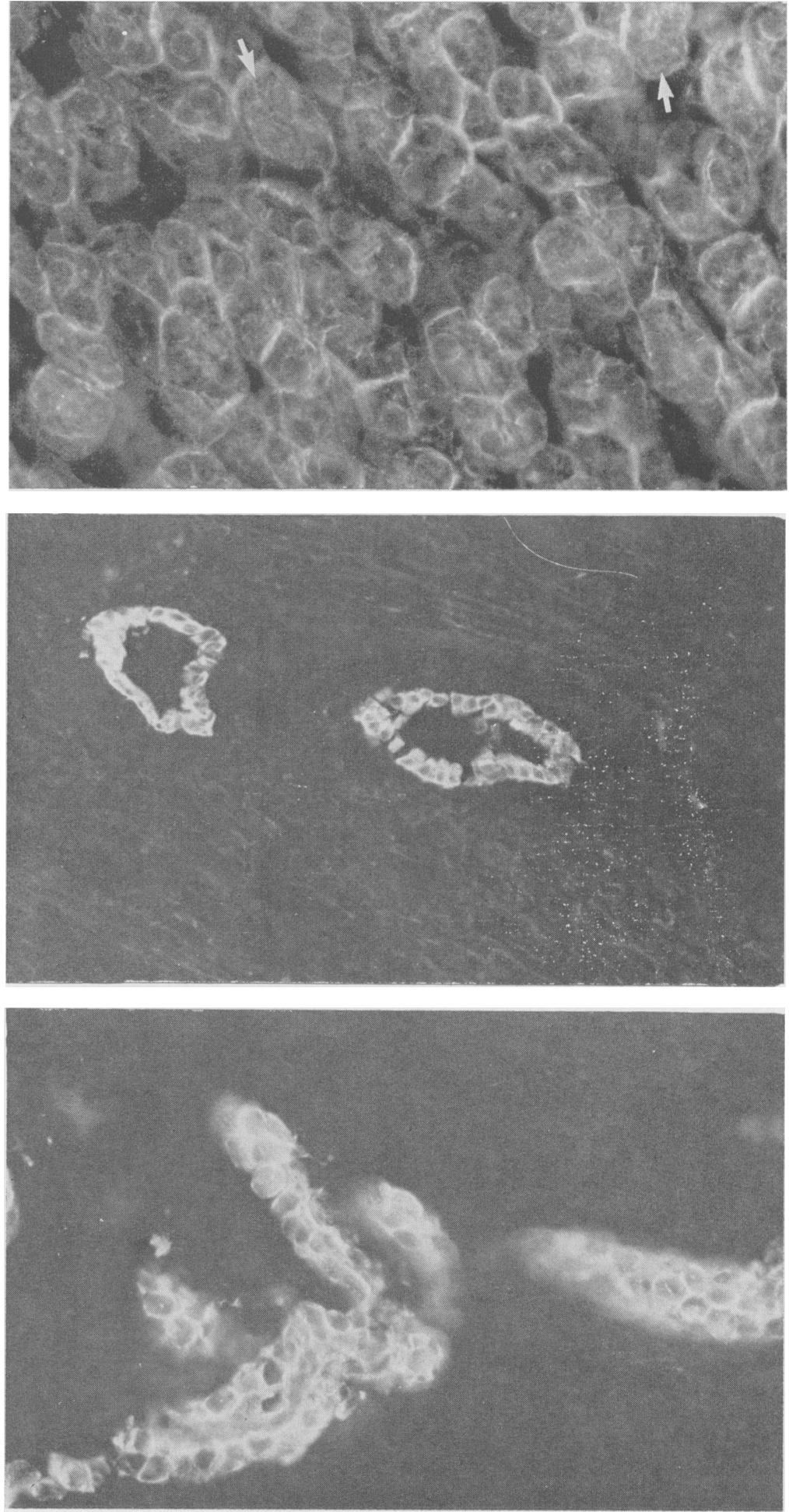


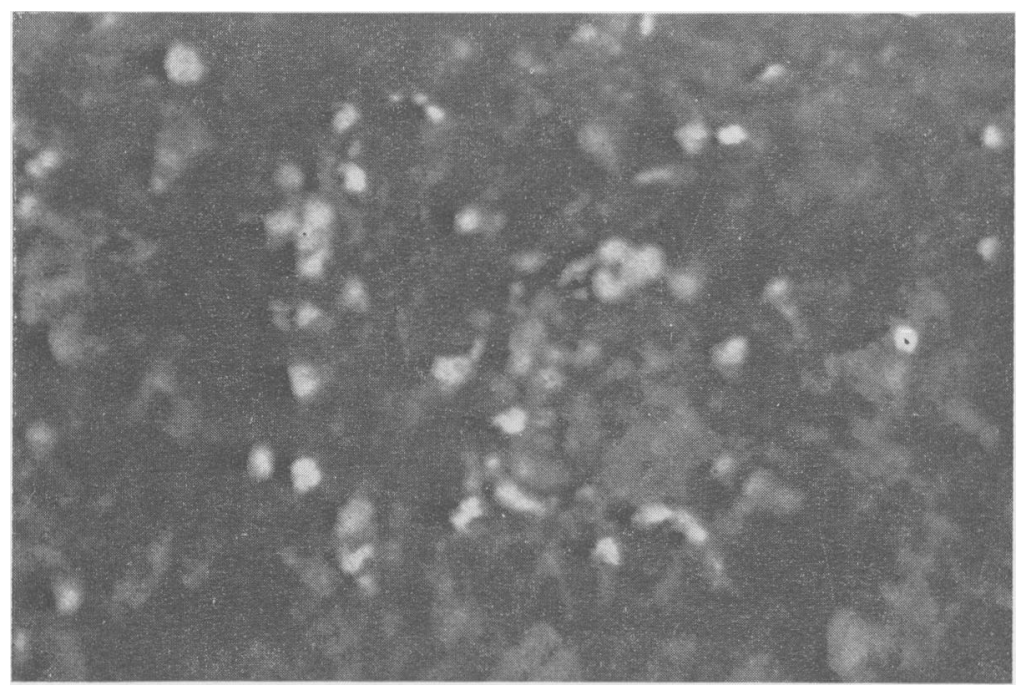

Fig. 6 Reaction of anti-JMB3 with alcoholic cirrhotic liver. Many 'mesenchymal' cells are labelled. The hepatocytes are entirely negative. $\times 400$.

not label MBs but reacted only with mesenchymal cells in the fibrous septa and sinusoids of alcoholic cirrhotic livers (Fig. 6); it did not react with any cell type in normal liver.

The antigens defined by antibodies produced by these three clones will subsequently be referred to as JMB1, JMB2, and JMB3 antigens and the antibodies themselves as anti-JMB1, anti-JMB2, and anti-JMB3. The specificities listed above were derived by testing each antibody on two alcoholic cirrhotic and seven normal livers.

Normal rabbit serum and the fluorescent reagents alone did not stain normal or alcoholic cirrhotic liver by the indirect immunofluorescence procedure. The monoclonal antibody to HLA (A, B, and C heavy chains) reacted only with vascular structures in the liver; these results will be reported in detail elsewhere (unpublished observations). ${ }^{13}$ These observations indicate that the reactivity of antiJMB1 and anti-JMB2 with MBs is not due to nonspecific antibody binding properties of MBs.

The reactivity of anti-JMB1, anti-JMB2, and rabbit antiserum to $\mathrm{MBP}^{7}$ were compared by immunofluorescence on three alcoholic cirrhotic livers containing MBs. In two cases (those referred to above) there was intense staining of MBs by both monoclonal antibodies but the MBs in the third case did not react. The MBs in all three livers reacted equally well with polyclonal rabbit antibody. All three of these livers, by routine pathological examination, were in all respects similar.

The effects of absorbing anti-JMB1, anti-JMB2, and anti-JMB3 with MBs, MBP, and normal liver homogenate are shown in the table. The ability of anti-JMB1 and anti-JMB2 to react with MBs is
Table Absorption of anti-JMB1, anti-JMB 2, and anti-JMB3 with $M B s, M B P$, and normal livers*

\begin{tabular}{|c|c|c|}
\hline Antibody & Absorbent & Staining reaction $\dagger$ \\
\hline Anti-JMB1 & $\begin{array}{l}\text { Nil } \\
\text { MBs } \\
\text { MBP } \\
\text { Normal liver }\end{array}$ & $\begin{array}{l}++t \\
-v e \\
+ \\
-t\end{array}$ \\
\hline Anti-JMB2 & $\begin{array}{l}\text { Nil } \\
\text { MBs } \\
\text { MBP } \\
\text { Normal liver }\end{array}$ & $\begin{array}{l}+ \\
\text {-ve } \\
+ \\
-v e\end{array}$ \\
\hline Anti-JMB3 & $\begin{array}{l}\text { Nil } \\
\text { MBs } \\
\text { MBP } \\
\text { Normal liver }\end{array}$ & $\begin{array}{l}+-+ \\
+++ \\
- \text { ve } \\
\text {-ve }\end{array}$ \\
\hline
\end{tabular}

* Monoclonal antibodies were absorbed as described in the Methods section.

tThe absorbed antibodies were tested by immunofluorescence on sections of an alcoholic cirrhotic liver containing MBs; the unabsorbed monoclonal antibodies-that is, absorbent $=$ nil-gave the staining reactions described in paragraph 1 of the Results section.

completely abolished by absorption with isolated MBs and markedly reduced by absorption with MBP. Normal liver did not absorb the reactivity of anti-JMB1 with MBs in tissue sections but completely abolished the reactivity of anti-JMB2 not only with MBs but with all cell membranes and cytoplasmic filaments. (It is probable that the entire reactivity of anti-JMB1 and anti-JMB2 for MBs could be completely absorbed by higher concentrations of MBP. MBs, on the other hand, are particularly effective absorbents, as they are particulate). The reactivity of anti-JMB3 for mesenchymal cells in alcoholic cirrhotic liver was unaffected by absorption with MBs but was completely abolished by absorption with MBP and normal liver. 


\section{Discussion}

Mallory bodies in alcoholic liver disease ${ }^{14}$ and in griseofulvin treated mice ${ }^{15}$ have a prominent filamentous component; the filaments have a diameter of $14-20 \mathrm{~nm} .{ }^{16}$ Because of this, several investigators have attempted to show that they are derived from one of the three cytoplasmic filamentous components of normal hepatocytes-namely, microfilaments, microtubules, and intermediate filaments. ${ }^{16}$ Microfilaments have a diameter of $4-6 \mathrm{~nm}$ and contain actin. The dimensions of these filaments and the fact that MBs do not contain actin ${ }^{76}$ argue against a derivation of MBs from this filament class. Similarly, it appears unlikely that MBs are composed of microtubules, as they are physically (mean diameter $22 \mathrm{~nm}$ ) and antigenically different from MBs; the former contain tubulin and the latter do not. ${ }^{16}$ As MBs contain prekeratin antigens, a component of epidermal cell intermediate filaments, and the fact that isolated MBs contain polypeptides similar in size to prekeratin(s) ${ }^{15}$ it has been suggested that MBs arise from a disturbance of intermediate filament metabolism. It seems unlikely, however, that MBs are solely composed of intermediate filament proteins, as they are physically larger than this filament class (diameter 6-11 nm). The results reported in this and a previous ${ }^{7}$ paper confirm this conclusion and demonstrate the antigenic complexity of MBs.

As shown here MBs contain a unique determinant - JMB1 - which is quite separate from the unique determinant defined by polyclonal antibodies to $\mathrm{MBP}^{7}$. JMB1 antigen can be demonstrated only in frozen sections, while the polyclonal antibody still recognises $\mathrm{MBs}$ in paraffin-embedded liver tissue $^{7}$ and, as shown here, the polyclonal antibody reacted with MBs in all three cases of alcoholic liver disease, while anti-JMB1 reacted only with MBs in two cases. The nature of these unique antigens is unknown but they may be conformational and dependent on the quaternary structure of MBs. This seems unlikely, as anti-JMB1 and the polyclonal antibody were produced against MBP, a reduced and denatured protein of apparent molecular weight of 53,000 daltons. ${ }^{7}$ It is possible that these apparently unique antigens are derived from a component or product of normal hepatocytes which is present in low concentration and cannot be detected by our present reagents and that it accumulates in hepatocytes damaged by alcohol.

MBs also contain a third antigen defined by monoclonal antibodies-JMB2. This antigen is present in a cytoplasmic filamentous system and is also associated with the cell and nuclear membranes of normal hepatocytes. JMB2 differs from prekeratin in that the latter cannot be demonstrated in normal liver cells by immunological procedures, although it is clearly present in MBs. ${ }^{6}{ }^{16}$ It has been shown, however, that intermediate filaments are closely associated with the plasma membranes of hepatocytes and can be found in small numbers scattered in their cytoplasm. ${ }^{16}$ It is possible, therefore, that anti-JMB2 detects a new intermediate filament antigen present in hepatocytes and bile duct epithelial cells. This conclusion has been substantiated by more recent work from this laboratory. ${ }^{17}$ It is interesting to note that JMB2 cannot be detected in the cell membranes or cytoplasmic filaments in cells which contain MBs. This observation probably indicates that the metabolism and/or secretion of this antigen is disrupted in these cells leading to its accumulation as part of MBs. This hypothesis is in some respects analogous to the concept of $\alpha-1$-antitrypsin accumulation in hepatocytes of patients with a genetic deficiency of this anti-proteinase..$^{18}$

The interpretation of the reactivity of the third monoclonal antibody-anti-JMB3-is not clear. As it does not react with MBs and only with mesenchymal cells of alcoholic cirrhotic liver, it could be directed against a contaminant in MBP. This explanation seems unlikely because the reactivity of anti-JMB3 for mesenchymal cells is completely abolished by absorption with MBP and normal liver but not by MBs themselves. The most likely interpretation of these observations is that the extraction of MBP from MBs, reveals a determinant which is occluded in intact MBs but is revealed in mesenchymal cells which have 'processed' MB after digestion. It would also follow that normal liver also contains JMB3 in concentrations which cannot be detected by our present reagents. Finally, the nature of the mesenchymal cells which contain JMB3 has not yet been determined and the characterisation of this antigen and its significance in alcoholic liver disease remain to be clarified.

In summary, it can be concluded from this paper that MBs contain unique determinants (defined by polyclonal antibodies to MBP); a second determinant-JMB1 - which may also be unique as it is not detectable in normal hepatocytes; a third determinant-JMB2-which may derive from an abnormality in intermediate filament metabolism. The significance of the JMB3 antigen for alcoholic liver disease remains to be clarified.

JB, KAF, and A McM thank the Wellcome Trust for grant support.

\section{References}

${ }^{1}$ Kendell RE. Alcoholism: a medical or a political problem? Br Med J 1979; 1 : 367-71. 
${ }^{2}$ Popper H. Alcohol and the liver. p. 289, Eds. Fisher MM, and Rankin JG; Pub 1. Plenum Press (New York), 1977.

${ }^{3}$ Zetterman RK, Luisada-Opper A, Leevy CM. Alcoholic hepatitis: cell mediated immunological response to alcoholic hyalin. Gastroenterology 1976; 70 : 382-4.

${ }^{4}$ Kanagasundurum N, Kakumu S, Chen T, Leevy CM. Alcoholic hyalin antigen (AHAg) and antibody (AHAb) in alcoholic hepatitis. Gastroenterology 1977; 73: 1368-73.

${ }^{5}$ Sim JS, Franks KE, French SW. Comparative electrophoretic study of Mallory body and intermediate filament protein. J Med 1978; 9: 211-21.

${ }^{6}$ Denk H, Franke WW, Eckerstorfer R, Schmid E, Kerkaschki D. Formation and involution of Mallory bodies (alcoholic hyalin) in murine and human liver revealed by immunofluorescence microscopy with antibodies to prekeratin. Proc Natl Acad Sci USA 1979; 76: 4112-6.

'Morton JA, Fleming KA, Trowell JM, McGee JO'D. Mallory bodies-immunohistochemical detection by antisera to unique non-prekeratin components. Gut 1980; 21 : 727-33.

${ }^{8}$ Nairn RC in Fluorescent protein tracing 4th ed. London: Churchill Livingstone, 1976.

${ }^{9}$ French SW, Ihrig TJ, Norum ML. A method of isolation of Mallory bodies in a purified fraction. Lab Invest 1972; 26: 240-4.

${ }^{10}$ Okamura K, Harwood TR, Yookoo H. Isolation and electrophoretic study on Mallory bodies from the livers of alcoholic cirrhosis. Lab Invest 1975; 33: 193-9.

${ }^{11}$ Kohler G, Milstein C. Continuous cultures of fused cells secreting antibody of predefined specificity. Nature 1975; 256: 445-7.

${ }^{12}$ Brodsky FM, Parham P, Barnstable CJ, Crumpton MJ, Bodmer WF. Monoclonal antibodies for analysis of the HLA system. Immunol Rev 1979; 47: 3-

${ }^{13}$ Submitted for publication.

${ }^{14}$ Yookoo H, Minick OT, Batti F, Kent G. Morphological variants of alcoholic hyalin. Am J Pathol 1972; 69: 25-40.

${ }^{15}$ Franke WW, Denk H, Schmid E, Osborn M, Weber K. Ultrastructural biochemical and immunological characterization of Mallory bodies in livers of griseofluvintreated mice. Lab Invest 1979; 40: 207-20.

${ }^{16}$ Franke WW, Schmid E, Kartenbeck J, Mayer D, Hacker H, Bannash P. et al. Characterisation of the intermediate sized filaments in liver cells by immunofluorescence and electron microscopy. Biol Cellulaire 1979; 34: 99-110.

${ }^{17}$ Morton JA, Bastin J, Fleming KA, McMichael A, Burns J, McGee J. O'D. A new intermediate filament antigen defined by monoclonal antibodies: Its involvement in alcoholic liver disease. (Submitted).

${ }^{18}$ Cox DW. Defect in $\alpha 1$ AT deficiency. Lancet, 1973; 2 : 844-45. 